\title{
Modulação da Contratilidade Cardíaca
}

\author{
Modulation of Heart Contractility
}

Tiago Luiz Silvestrini ${ }^{1 *}$, Rafael de March Ronsoni ${ }^{1}$, Celso Salgado ${ }^{2}$

ORCID IDs

Silvestrini TL (D) https://orcid.org/0000-0002-5714-7308

Ronsoni RM (D) https://orcid.org/0000-0001-7135-9844

Salgado C (D) https://orcid.org/0000-0002-2715-2448

\section{RESUMO}

Pacientes com insuficiência cardíaca (IC) vêm se beneficiando da terapia elétrica por meio de marcapassos convencionais quando associada à bradicardia e à terapia de ressincronização cardíaca ou com fração de ejeção rebaixada e presença de QRS maior que 150 ms, principalmente na presença de bloqueio de ramo esquerdo. Outros grupos de pacientes com IC apresentam limitações ao tratamento com eletroterapia. No entanto, um conceito antigo tem tomado espaço no tratamento de um grupo de pacientes que fica fora das diretrizes nacionais e internacionais para eletroterapia na IC: a modulação da contração cardíaca. Este artigo tem como objetivo apresentar a revisão das evidências científicas já produzidas e publicadas acerca dessa nova modalidade de tratamento da IC.

PALAVRAS-CHAVE: Insuficiência cardíaca; Marcapasso artificial; Terapia por estimulação elétrica.

\begin{abstract}
Patients with heart failure (HF) are being benefited by electric therapy through conventional pacemakers when associated to bradycardia and cardiac resynchronization therapy or with low ejection fraction and presence of QRS longer than 150 ms, mainly in the presence of left branch block. Other groups of patients with HF present limitations regarding electrotherapy. However, an old concept has gained space in the treatment of patients who are outside the national and international guidelines for electrotherapy in HF: the modulation of heart contractility. This article has the purpose of presenting a review of already produced scientific evidence regarding this new modality for HF treatment.
\end{abstract}

KEYWORDS: Heart failure; Artificial pacemaker; Electric stimulation therapy.

1.Instituto de Ritmologia Cardíaca - Itajaí/SC - Brasil.

2.Universidade Federal do Triângulo Mineiro - Serviço de Marcapasso - Uberaba/MG - Brasil.

*Autor correspondente: tlsilvestrini@hotmail.com

Recebido: 30 Set 2018 | Aceito: 5 Jul 2019

Editor Associado: José Mario Baggio Junior 


\section{INTRODUÇÃO}

A eletroterapia vem auxiliando o tratamento de pacientes com insuficiência cardíaca (IC) desde a década de 1990 e solidificou-se como opção no arsenal terapêutico para esses pacientes. $\mathrm{Na}$ década passada, importantes estudos demonstraram o benefício na qualidade de vida, na morbidade e na mortalidade da terapia de ressincronização cardíaca (TRC) e na redução da mortalidade pelo uso dos cardiodesfibriladores implantáveis $(\mathrm{CDIs})^{1,2}$.

Com relação à melhora da qualidade de vida e dos parâmetros clínico, laboratorial e hemodinâmico, a TRC continua sendo a única opção terapêutica a ser utilizada para a maioria dos pacientes, quando houver indicação para implante de algum dispositivo artificial de estimulação cardíaca.

Porém, atualmente, apenas um grupo muito limitado de pacientes pode se beneficiar dessa terapia: aqueles com miocardiopatia dilatada com disfunção grave do ventrículo esquerdo (VE), QRS com duração maior que $130 \mathrm{~ms}$ (principalmente maior que $150 \mathrm{~ms}$ ) e com sintomas associado a IC ${ }^{2}$.

A modulação da contratilidade cardíaca (MCC) por meio do implante de estimulador cardíaco artificial vem apresentando benefícios na qualidade de vida, teste de caminhada de seis minutos e parâmetros hemodinâmicos em um grupo de pacientes no qual a TRC não tem indicação convencional, como aqueles com sintomas incapacitantes, com QRS com duração menor que $130 \mathrm{~ms}$ e fração de ejeção (FE) entre 25 e $45 \%{ }^{3}$.

\section{DISPOSITIVO IDEALIZADO PARA MODULAÇÃO DA CONTRATILIDADE}

Trata-se de um dispositivo com estrutura semelhante ao de um marcapasso cardíaco convencional. Existem três conexões, uma para o cabo-eletrodo atrial e duas para os cabos-eletrodos ventriculares a serem implantados no septo interventricular do ventrículo direito (VD). Os cabos-eletrodos são os mesmos da estimulação cardíaca convencional. $\mathrm{O}$ dispositivo tem um carregador externo de bateria utilizado para recarregá-la em média uma vez a cada três ou quatro semanas e um programador específico (Fig. 1) ${ }^{3}$.

O implante do dispositivo é muito semelhante ao de um marcapasso convencional, com o cabo-eletrodo atrial implantado no apêndice atrial ou parede lateral e os caboseletrodos ventriculares no septo interventricular na região médio-septal, com distância mínima de $2 \mathrm{~cm}$ entre as pontas desses cabos-eletrodos. Os testes realizados são os mesmos para avaliação de cabos-eletrodos convencionais, nos quais se busca, como melhor parâmetro, uma boa sensibilidade do sinal ventricular ${ }^{3}$ (Figs. 2 e 3 ). (a)

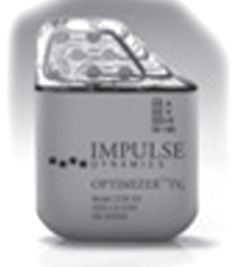

(b)

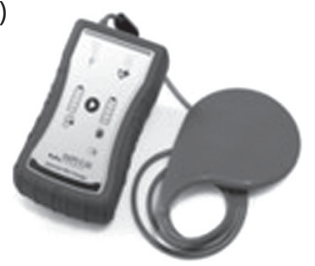

(c)

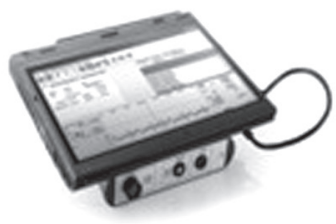

Figura 1. (a) gerador de pulsos Optimizer IVs; (b) Carregador externo utilizado para recarga da bateria e (c) programador do dispositivo.

(a)

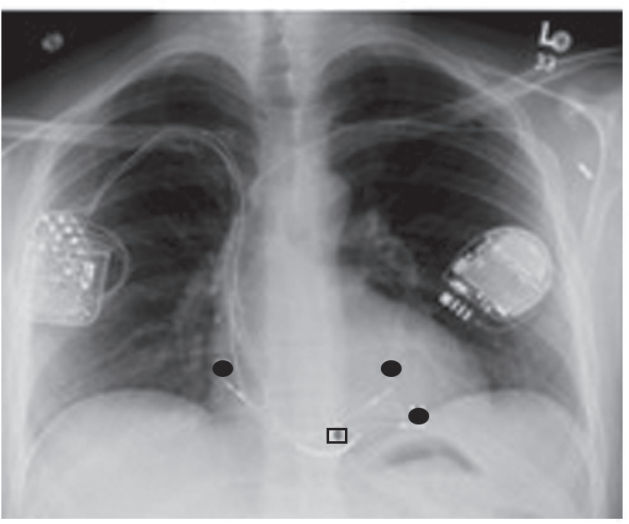

(b)

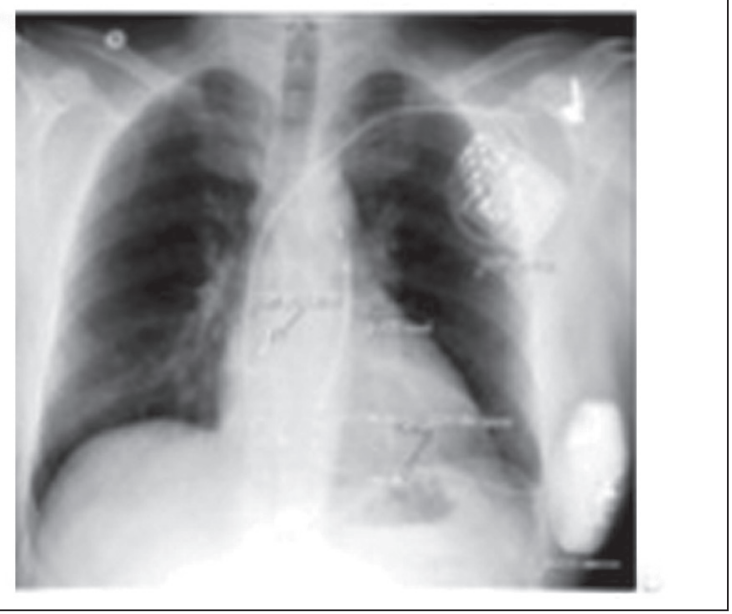

Figura 2. (a) radiografia pós-implante de paciente com CDI unicameral implantado a esquerda e MCC implantado a direita (círculos pretos demonstrando as pontas dos caboseletrodos do MCC e quadrado preto demonstrando a ponta do cabo-eletrodo do CDI). (b) radiografia pós implante de CDI subcutâneo e MCC, ambos em hemitórax esquerdo. 


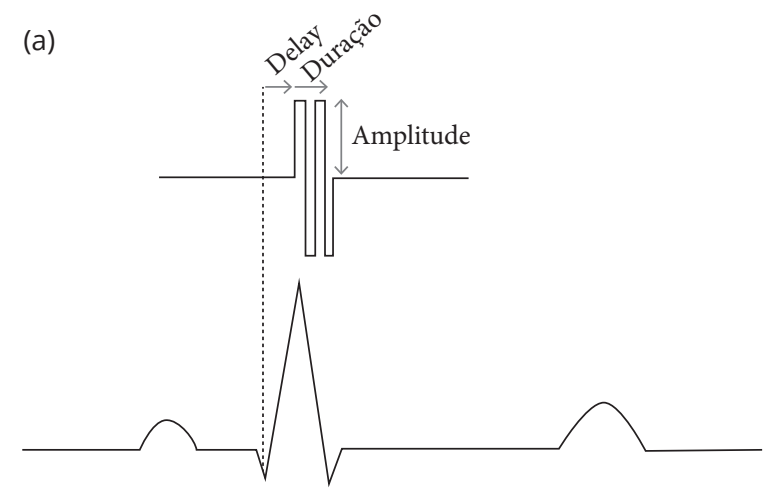

(b)

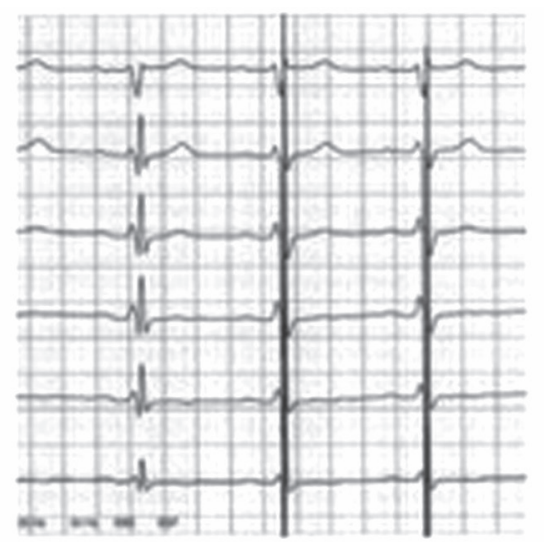

Figura 3. (a) Desenho demonstrando os sinais bifásicos liberados pelo MCC após atraso pré-estabelecido para deflagrar durante o período refratário ventricular e desta maneira não provocar excitação ventricular. (b) ECG de superfície demonstrando um batimento antes do início da MCC e depois 2 batimentos após aplicação.

A MCC é realizada por meio da liberação de sinal bifásico de alta voltagem de 7,5 v com $22 \mathrm{~ms}$ de duração durante o período refratário absoluto ${ }^{3}$.

\section{FISIOLOGIA DO CÁLCIO NO CICLO DE CONTRATILIDADE-RELAXAMENTO CARDÍACO}

Para compreender o mecanismo de ação desse novo dispositivo, é importante relembrar o papel do ciclo do cálcio no acoplamento excitação-contratilidade do músculo cardíaco. Isso tem especial importância na fase aguda e subaguda da ação da MCC.

O cálcio tem papel crucial na regulação das fases de contratilidade e relaxamento do músculo cardíaco. A associação entre os fluxos de cálcio e a ligação da contratilidade com a onda de excitação (acoplamento excitação-contração) estão razoavelmente bem compreendidas. A principal hipótese está relacionada à liberação de cálcio do retículo sarcoplasmático $(\mathrm{RS})^{4,5}$.

Pequenas quantidades de cálcio entram e saem do cardiomiócito a cada ciclo cardíaco via membrana sarcoplasmática, e maior quantidade de cálcio chega ao citoplasma da célula a partir do RS (Fig. 4).

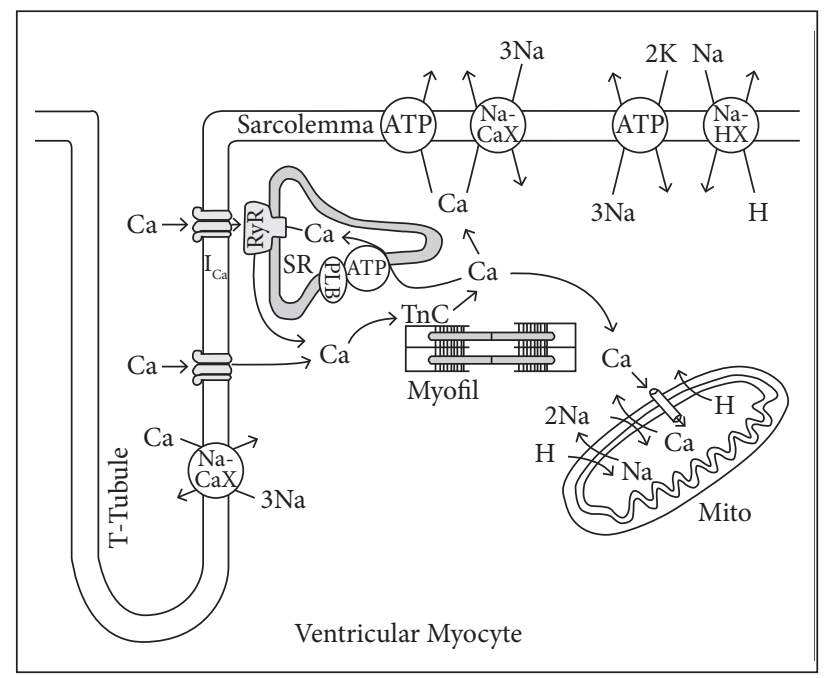

Figura 4. Diagrama esquemático dos fluxos de cálcio dentro do miócito durante o ciclo cardíaco.

Cada onda de despolarização cardíaca que percorre os miócitos pelos túbulos $\mathrm{T}$ abrem os canais de cálcio tipo $\mathrm{L}$ da membrana citoplasmática, próximos ao RS, ativando, assim, os canais de liberação de cálcio, chamados receptores ryanodine. A despolarização do miócito, portanto, libera relativamente grande quantidade de cálcio dentro do citosol em resposta à pequena entrada no cardiomiócito oriunda da despolarização. Esse processo eleva em 10 vezes a concentração de íon cálcio dentro do citosol. $\mathrm{O}$ resultado é o aumento da interação de íons cálcio com troponina C para iniciar o processo de contratilidade ${ }^{4,5}$.

\section{CANAIS DE LIBERAÇÃO DE CÁLCIO DO RS Receptores Ryanodine}

Cada canal de cálcio tipo L do sarcolema controla um grupo de seis a 20 canais de liberação do RS por proximidade anatômica dos canais de cálcio dos túbulos $\mathrm{T}$ com os canais liberadores situados no RS. O canal liberador de cálcio é parte de uma complexa estrutura chamada receptor ryanodine ${ }^{4}$.

Os receptores ryanodine têm duas funções: 1) controlar os canais liberadores de cálcio do RS; e 2) atuar como arcabouço que contém uma série de proteínas-chave reguladoras para o complexo juncional. Essas proteínas incluem aquelas que 
respondem pela fosforilação por meio da proteína kinase $\mathrm{A}$ (PKA) e sua proteína ancoradoura AKAP4.

A inativação da liberação de cálcio pelo RS após seu aumento dentro do citosol não é bem compreendida e existem várias hipóteses para esse fenômeno: 1) o aumento da concentração de cálcio no citosol inibe o processo de liberação de mais cálcio; 2) o aumento da concentração de cálcio no citosol pode ativar uma bomba de captação de cálcio pelo RS; 3) o RS torna-se depletado de cálcio; e 4) o receptor ryanodine torna-se inativado, ficando resistente à concentração de cálcio. Independentemente do modo que ocorre, a queda da concentração de cálcio no citosol provoca o início da diástole ${ }^{4}$.

\section{CAPTAÇÃO DE CÁLCIO PELO RS POR MEIO DA CÁLCIO ATPase}

Os íons cálcio são recaptados para dentro do RS por uma bomba de cálcio chamada SERCA que tem várias isomorfas, sendo a predominante no coração a SERCA2 $\mathrm{a}^{4,5}$. Para cada ATP hidrolisado por essa enzima, dois íons cálcio são recaptados para dentro do RS. A fonte de energia é oriunda da geração de ATP do citosol pela glicólise. Importantes ligações entre a SERCA e a contratilidade cardíaca são encontradas. Por exemplo, na IC, a atividade da SERCA está diminuída $\mathrm{a}^{4,5}$.

Phospholamban é o nome dado para os "receptores de fosfato", e sua atividade é regida por um estado de fosforilação, processo que altera a configuração molecular da SERCA para promover sua atividade. Duas proteínas kinases maiores estão envolvidas nesse processo: uma é ativada pela $\mathrm{PKA}$ em resposta à estimulação beta-adrenérgica e AMP cíclico e outra pelos íons de cálcio e calmodulin que atuam em dois diferente sítios de fosforilação. Quando a phospholamban responde à estimulação beta-adrenérgica do cardiomiócito por ganho da recaptação de cálcio pela SERCA dentro do retículo RS, aumentando a taxa de relaxamento, a maior ativação é a fosforilação do sítio da PKA. Adicionalmente, o aumento de estoques de cálcio dentro do RS corresponde ao aumento da quantidade de cálcio liberada pelo receptor ryanodine; em resposta, a subsequente onda de despolarização gera aumento na frequência e força de contração ${ }^{4}$.

O cálcio, recaptado para dentro do RS pela bomba de recaptação de cálcio, fica aí estocado antes da próxima liberação. A calcequestrina, proteína que estoca o cálcio no
RS, fica na região do RS próximo aos túbulos T. O cálcio estocado pela calcequestrina torna-se disponível para o processo de liberação assim que essa descarrega cálcio dentro da entrada interior do seu canal de liberação. Esse processo troca os íons cálcio liberados da entrada externa para dentro do citosol. A calreticulin é outra proteína estocadora de cálcio com estrutura e função similares à calcequestrina $a^{4-6}$.

\section{CONTROLE DO SARCOLEMA DOS ÍONS CÁLCIO E SÓDIO Canais de Cálcio}

O início do processo de excitação-contração ocorre pela abertura dos canais de cálcio tipo L na membrana sarcoplasmática. Esse canal é altamente seletivo ao cálcio e permite sua transferência para dentro do citosol no seu estado aberto, quando ocorre a despolarização da membrana ${ }^{4}$.

Após ser ativado (aberto) pela despolarização da membrana, o canal de cálcio é inativado (volta a ficar fechado) pelo: 1) aumento da voltagem durante a despolarização em um potencial mais positivo do que durante a ativação; e 2) aumento da concentração interna de cálcio, especialmente o fluxo de cálcio do receptor ryanodine que empurra a concentração desse, presente no espaço subsarcolemal interno, para próximo da entrada dos canais $\mathrm{L}$ dos tubos $\mathrm{T}$ para ajudar a terminar o fluxo de corrente ${ }^{4,5}$.

\section{Bombas de Íons e Trocadores de Íons}

Para contrabalancear a entrada de cálcio dentro da célula cardíaca a cada ciclo, a mesma quantidade deve sair por um dos dois processos: 1) o cálcio pode ser trocado por sódio pela troca sódio-cálcio; e 2) pela bomba de cálcio por consumo de ATP que o transfere para fora da célula contra um gradiente de concentração ${ }^{4}$.

$\mathrm{Na} \mathrm{IC}$, as mudanças no ciclo do cálcio são fundamentais para a piora da contratilidade do músculo cardíaco. Os estoques de cálcio da RS são severamente depletados devido à combinação de efeitos negativos: 1) diminuição da atividade da SERCA; e 2) fuga de cálcio na fase diastólica associada à hiperfosforilação e função anormal do receptor ryanodine. Essas alterações provocam depleção de cálcio no RS, diminuição da liberação no RS, escape diastólico de cálcio e aumento desse no citosol. Além disso, a ação dos canais de cálcio tipo L estão reguladas para baixo ${ }^{4}$. 


\section{MECANISMO DE AÇÃO DO MCC}

A ação da MCC está relacionada ao manejo do ciclo do cálcio na fase aguda. Cronicamente, a melhora da contratilidade cardíaca ocorre pelo incremento na fosforilação das vias regulatórias das chaves de cálcio, o que aumenta a contratilidade e restaura o padrão normal, que é o perfil do gene fetal da $\mathrm{IC}^{3}$.

\section{Aumento Agudo da Contratilidade}

Estudos em faixas de tecidos de músculos papilares de coelhos e cães com IC produzida por embolização coronariana e músculo trabecular de pacientes com IC demonstram aumento da contratilidade e da FE com início da estimulação com modulador da contratilidade cardíaca $^{7,8}$.

Entre os benefícios do ciclo do cálcio provocado pela MCC estão a regulação para cima dos canais de cálcio tipo $L$ e a melhora da captação do cálcio dentro do RS, provocando aumento do influxo extracelular durante a contratilidade subsequente ao início da modulação e a liberação de cálcio desse retículo ${ }^{3}$ (Fig. 5).

\section{EFEITOS CRÔNICOS NA IC}

A modulação demonstrou melhora da $\mathrm{FE}$, no volume sistólico, $\mathrm{dP} / \mathrm{dT}$ de $\mathrm{VE}$ e retardo no aumento do volume diastólico e sistólico final do VE e do VD? .
A IC produz mudanças no fenótipo do cardiomiócito para um padrão mais juvenil via reversão para um programa de gene fetal. Dessa forma, há aumento da expressão de BNP (brain natriuretic peptide) e do trocador cálcio-sódio, com diminuição da expressão de SERCA2A, alpha-MHC (major histocompatibility complex) e phospholamban. O uso crônico de MCC em animais com IC causa remodelamento reverso do programa de gene fetal em direção, novamente, ao programa normal adulto $^{10,11}$. Dessa forma, o ciclo do cálcio dentro do cardiomiócito é melhorado.

A regulação para cima da SERCA e a maior fosforilação da phospholamban aumentam a captação de cálcio pelo RS, resultando em maior liberação no batimento subsequente e, portanto, aumentando a contratilidade cardíaca.

Estudos demonstram que essas ações na mudança da expressão dos genes na regulação do cálcio nos cardiomiócitos são vistas duas horas após o início da estimulação, localmente, nos cardiomiócitos próximos à ponta do cabo-eletrodo ${ }^{14}$. Porém, após três meses de MCC, sítios distantes também demonstram os mesmos benefícios. Isso representa uma reversão geral da fisiopatologia da expressão do gene fetal na $\mathrm{IC}^{11,12}$.

\section{Estudos Clínicos}

Mais de 3.000 pacientes ao redor do mundo receberam implante dos dispositivos de MCC. Vários estudos clínicos vêm confirmando os bons resultados dos estudos pré-clínicos e

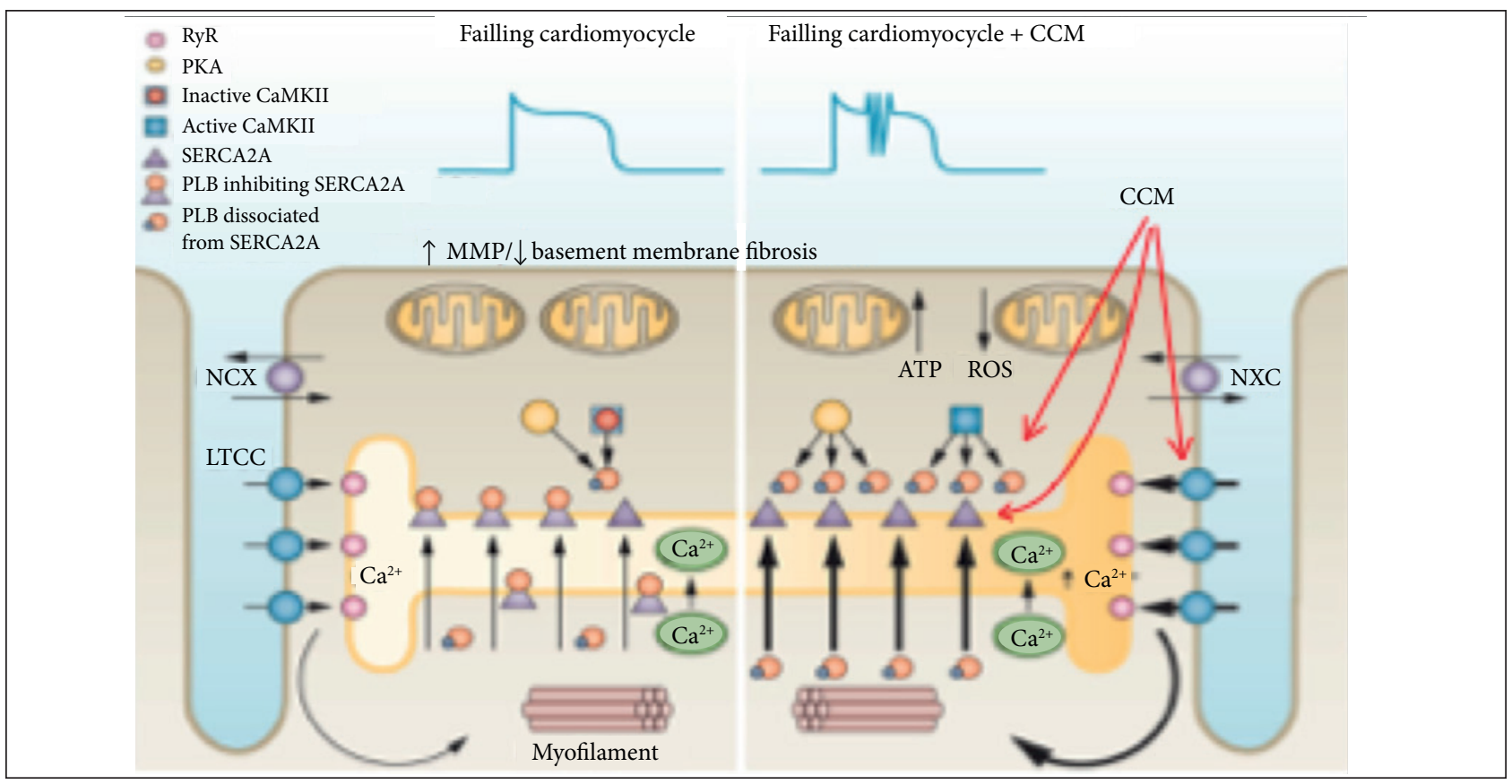

Figura 5. Diagrama esquemático do fluxo de cálcio no miocárdio doente (à direita) e do mecanismo de ação da MCC (à esquerda). 
apontando na direção de que a MCC poderá trazer benefício além da terapia médica otimizada para $\mathrm{IC}^{3}$.

No primeiro grande estudo clínico em humanos, o FIX-HF-3, publicado em 2004, 25 pacientes, com idade média de 62 anos, submeteram-se ao implante de modulador da contratilidade cardíaca, com IC da classe funcional da New York Heart Association (CF NYHA) III, refratários ao tratamento medicamentoso otimizado. Ainda como critérios de inclusão, selecionaram-se pacientes com FE abaixo de 35\% e largura de QRS menor ou igual a $130 \mathrm{~ms}$. Doze pacientes tinham como doença de base miocardiopatia idiopática e 13 doença coronariana. A avaliação aguda foi dada pela $\mathrm{dP} / \mathrm{dT}$. Após implante, o gerador foi ativado três horas por dia por oito semanas ${ }^{13}$.

Em 23 de 25 pacientes, o dispositivo foi implantado com sucesso. Houve melhora significante da CF NYHA de III para II em 15 pacientes e para I em 4 pacientes. A FE subiu de 22 para $28 \%$ e o escore de qualidade de vida para pacientes com IC de Minnesota Living with Heart Failure Questionnaire $(M L H F Q)$ melhorou de 43 pontos para 25 pontos. $O$ teste de caminhada de seis minutos subiu de $411 \mathrm{~m}$ para $465 \mathrm{~m}^{13}$.

Com relação a eventos adversos, nove pacientes apresentaram algum desconforto intermitente com a estimulação. Houve duas mortes não relacionadas ao dispositivo. Schmidinger et al. ${ }^{13}$, em posse desses resultados, concluíram que a MCC é uma técnica promissora para melhorar a função sistólica e sintomas de pacientes com IC refratária ao tratamento medicamentoso otimizado ${ }^{13}$.

Esses resultados impulsionaram a condução de um estudo clínico maior, o FIX-HF-4, publicado em 2008. Foram incluídos pacientes com IC sintomática (CF NYHA maior ou igual a II), de origem idiopática ou isquêmica, $\mathrm{FE}<35 \%$ e pico de captação de oxigênio $\left(\mathrm{VO}_{2 \max }\right)$ entre $10 \mathrm{e}$ $20 \mathrm{~mL} 0_{2} / \mathrm{min} / \mathrm{Kg}$. Os pacientes estavam em uso de máxima medicação tolerada para IC. Excluíram-se os pacientes com indicação para TRC convencional, com fibrilação atrial, infarto agudo do miocárdio no período de três meses antes da randomização, outras modalidades de doença coronariana, IC descompensada e arritmias ventriculares frequentes ${ }^{14}$.

Randomizaram-se 164 pacientes em dois grupos (1 e 2) durante dois períodos (fases 1 e 2), por 12 semanas em cada fase ${ }^{14}$.

No final de cada fase, realizou-se o seguinte protocolo: teste de estresse cardiopulmonar $\left(\mathrm{com} \mathrm{VO}_{2 \max }\right.$ ), MLHFQ, teste de caminhada de seis minutos e avaliação da CF NYHA ${ }^{14}$.
Os endpoints primários foram as medidas do $\mathrm{VO}_{2 \max } \mathrm{e}$ o MLHFQ ao final de cada fase, em cada grupo. Endpoints secundários foram mudanças da $\mathrm{CF}$ e teste de caminhada de seis minutos ${ }^{14}$.

O grupo 1 foi composto de 80 pacientes que inicialmente receberam o dispositivo ligado, enquanto o grupo 2 foi composto de 84 pacientes com dispositivo desligado. $\mathrm{Na}$ fase 2, os grupos receberam o tratamento oposto ao inicial ${ }^{14}$.

Durante a primeira fase, $\mathrm{VO}_{2 \max }$ aumentou similarmente em ambos os grupos; no entanto, na segunda fase, $\mathrm{o} \mathrm{VO}_{2 \max }$ continuou aumentando no grupo com tratamento ativo e diminuiu no grupo do dispositivo desligado ${ }^{14}$.

O MLHFQ melhorou em ambos os grupos na primeira fase, sendo maior no grupo com tratamento ativo, e continuou melhorando após cross-over no grupo em que o aparelho estava ligado, enquanto piorou no grupo em que o aparelho estava desligado ${ }^{14}$.

O teste de caminhada teve comportamento semelhante ao resultado do $\mathrm{VO}_{2 \max }$ e a avaliação da $\mathrm{CF}$ teve melhora em ambos os grupos durante as duas fases ${ }^{17}$ (Fig. 6).

Os autores concluíram que houve melhora consistente na tolerância ao exercício e qualidade de vida com MCC.

$\mathrm{O}$ estudo FIX-HF-5 foi o maior estudo realizado para avaliar a extensão da eficácia e a segurança do MCC. Foi realizado em 50 centros nos Estados Unidos da América e incluiu 428 pacientes CF NYHA III/IV, com QRS estreito e $\mathrm{FE} \leq \mathrm{a} 35 \%$ que foram randomizados para receber tratamento médico otimizado (TMO) (213 pacientes) versus TMO+MCC (215 pacientes). O endpoint primário de eficácia foi o limiar de ventilação anaeróbico e os secundários foram $\mathrm{VO}_{2 \max } \mathrm{e}$ MLHFQ após seis meses. O endpoint primário de segurança foi o teste de não inferioridade entre os grupos em 12 meses para o composto de todas as causas de mortalidade e hospitalização ${ }^{15}$.

Com relação ao endpoint composto de segurança, o grupo TMO apresentou 103 eventos nos 213 pacientes $(48,4 \%)$ e o grupo TMO+MCC 112 eventos dentre os 215 pacientes $(52,1 \%)$. Essa diferença ficou dentro do limite preestabelecido, encontrando, assim, endpoint de segurança para o tratamento com $\mathrm{MCC}^{15}$.

Quanto aos resultados de eficácia, o limiar de ventilação anaeróbico (endpoint primário de eficácia) diminuiu em ambos os grupos em 0,14 mL/kg.min após 24 semanas. O VO2max aumentou no grupo TMO+MCC e diminuiu no grupo TMO, com diferença encontrando significância estatística. O MLHFQ e NYHA melhoram mais significativamente no grupo TMO+MCC do que no grupo TMO. Houve 
também melhora não significativa no teste de caminhada de seis minutos no grupo $\mathrm{TMO}+\mathrm{MCC}^{15}$.

Esse estudo conseguiu encontrar o endpoint primário de segurança, porém não atingiu o endpoint primário de eficácia, que era a melhora no limiar de ventilação anaeróbico. Entretanto, houve melhora no $\mathrm{VO}_{2 \max }$ e na MLHFQ, assim como na CF da NYHA. Esses dois últimos achados foram semelhantes aos encontrados em estudo que validou a utilização do uso dos dispositivos de $\mathrm{TRC}^{15}$.

$\mathrm{Na}$ análise de subgrupos, percebeu-se que pacientes em NYHA III e FE acima de 25\% obtiveram os melhores resultados ${ }^{15}$ (Fig. 7).

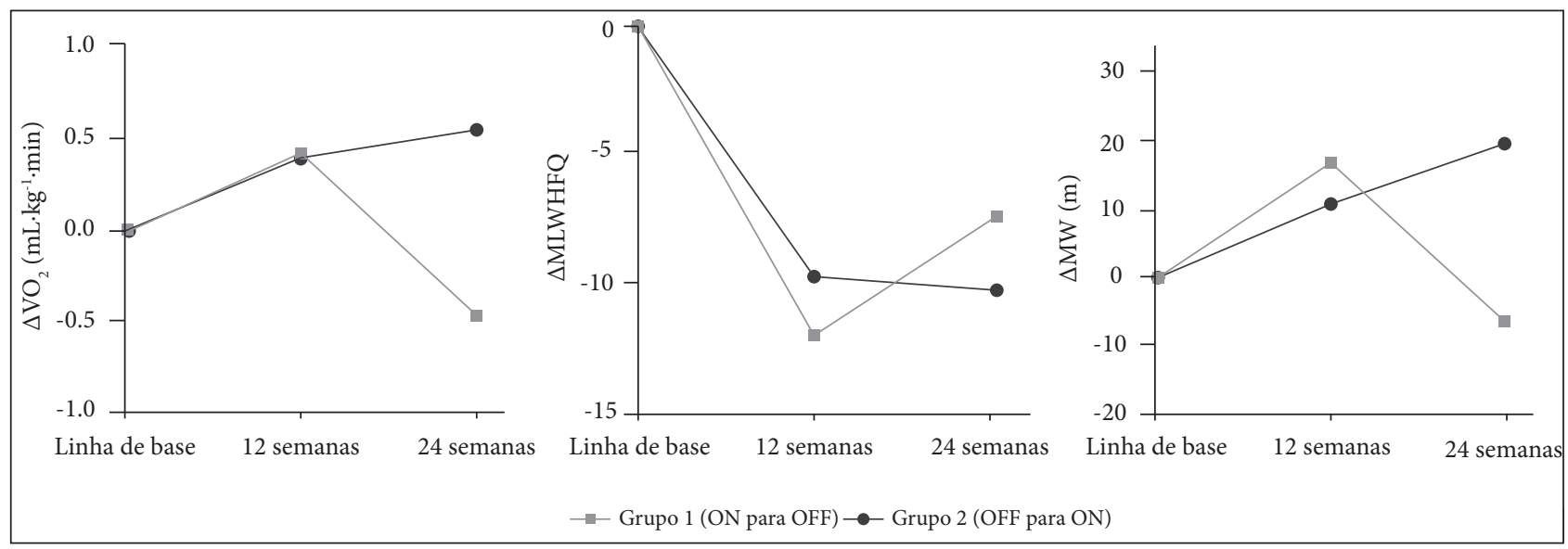

Figura 6. (a) Mudanças no $\mathrm{VO}_{2 \mathrm{max}}$ em cada grupo em comparação com seus respectivos valores de linha de base. Os resultados apresentados para os casos com dados completos; esses resultados concordam substancialmente com aqueles baseados em múltiplas atribuições. (b) Mudanças no Minnesota Living with Heart Failure Questionnaire em cada grupo em comparação com seus respectivos valores basais. Resultados apresentados para os casos com dados completos; esses resultados concordam substancialmente com aqueles baseados em múltiplas atribuições. (c) Mudanças no teste de caminhada de 6 minutos em cada grupo em comparação com seus respectivos valores basais ${ }^{17}$.

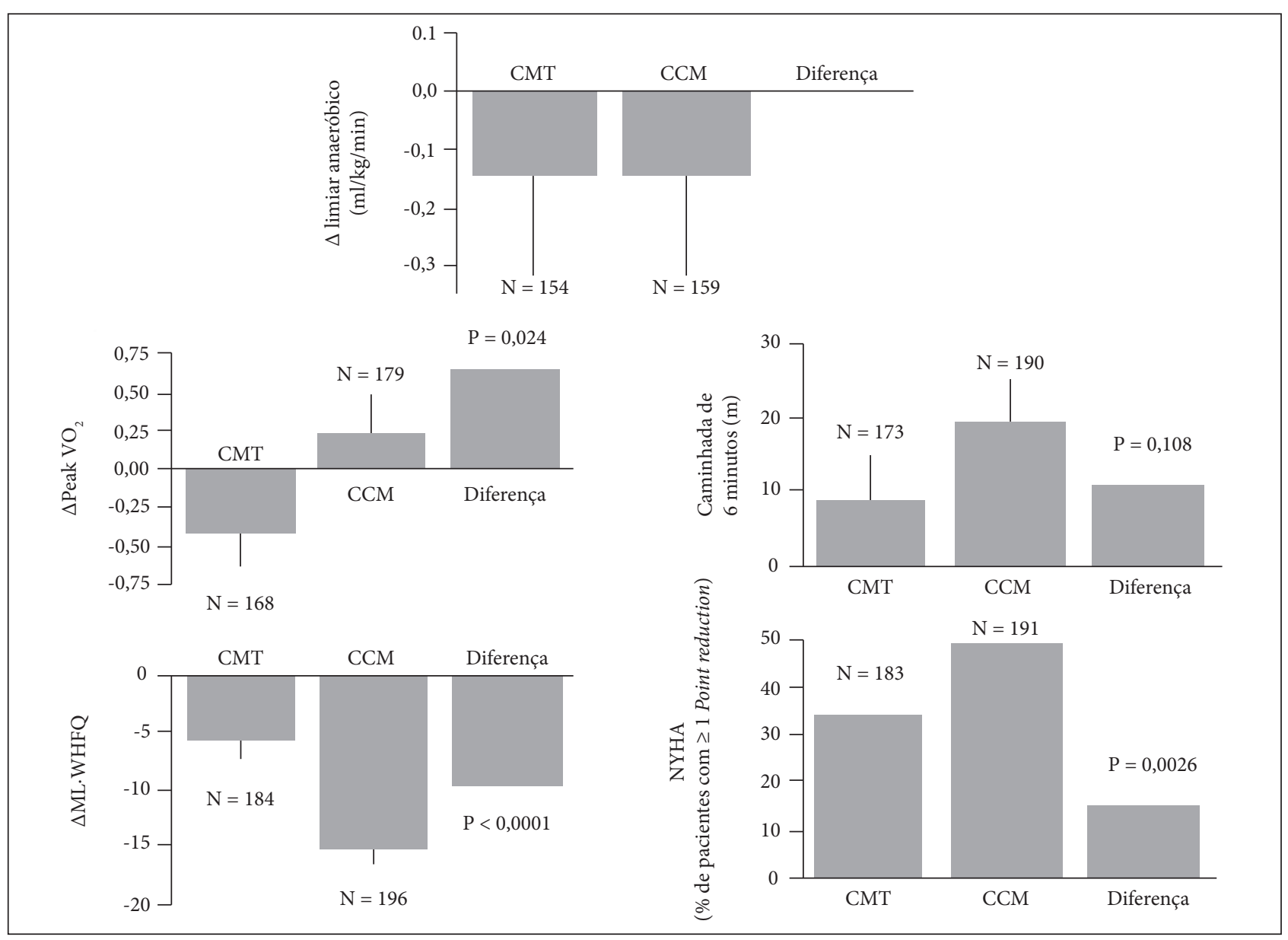

Figura 7. Gráficos com o resumo dos resultados do estudo FUX-HF-5. 
A utilização desse endpoint primário (limiar de ventilação anaeróbio) para este estudo foi requerida pelo Food and Drug Administration (FDA), visto que se tratava de estudo não cego e medidas como qualidade de vida e tolerância aos exercícios são parâmetros subjetivos, sucessíveis ao efeito placebo $^{15}$.

Os autores criticaram, dentre outros aspectos, esse fato e alegaram que, embora o limiar de ventilação anaeróbio seja um parâmetro objetivo de avaliação, não tem sido validado como endpoint em estudos de $\mathrm{IC}^{15}$.

Duas meta-análises sobre o tema foram publicadas. Em 2012, Cheuk-Man et a ${ }^{16}$. publicaram uma meta-análise sobre estudos controlados registrados no Cochrane, MEDLINE e EMBASE, comparando MCC com TMO ou tratamento sham. Os resultados de interesse foram todas as causas de mortalidade, todas as causas de hospitalização e efeitos adversos. Três estudos randomizaram 641 pacientes e a análise dessa população demonstrou que, comparado com controle, a MCC não diminuiu de forma estatisticamente significativa a mortalidade e a hospitalização e, em contrapartida, também não aumentou a risco de efeitos adversos ${ }^{16}$.
Outra meta-análise, publicada em 2014 por Giallauria et al. ${ }^{17}$, tendo como base de dados a mesma população da publicação anterior, utilizaram os endpoints primários de $\mathrm{VO}_{2 \max }$, teste de caminhada de seis minutos e qualidade de vida aferida pelo questionário MLHFQ. Essa análise conclui que, comparada ao tratamento padrão para IC, a MCC melhorou significativamente o $\mathrm{VO}_{2 \max }$, a distância percorrida no teste de seis minutos e a qualidade de vida aferida pelo MLHFQ(Fig. 8).

\section{Resultados em Longo Prazo}

Quatro publicações apresentaram resultados em longo prazo na mortalidade dos pacientes tratados com MCC. Schau et al. ${ }^{21}$ avaliaram retrospectivamente 54 pacientes submetidos a implante de MCC entre 2003 e 2010. Os pacientes apresentavam disfunção ventricular moderada a severa, NYHA III/IV e FE média de 23\%. Em seguimento de três anos, 24 pacientes faleceram (18,4\% por ano). A mortalidade foi equivalente à previsão esperada para essa população pelo modelo de predição de mortalidade para IC de Seattle Heart Failure Model (SHFM).

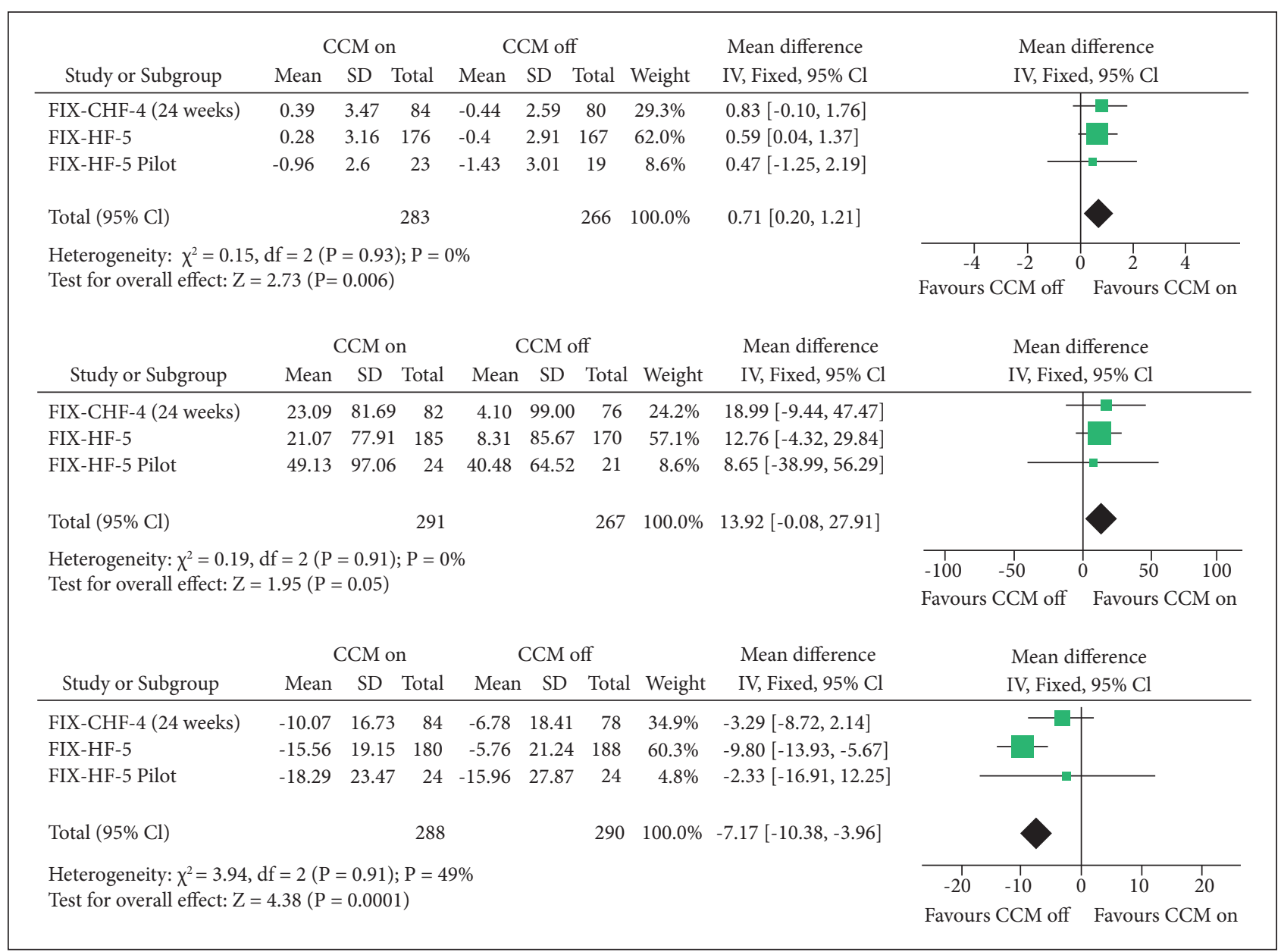

Figura 8. Tabelas dos resultados das mudanças no $\mathrm{VO}_{2 \max }$ (tabela superior). 
Em outro estudo, publicado por Kuschyk et al. ${ }^{19}$ e conduzido em um único centro, 81 pacientes foram acompanhados por três anos, entre 2004 e 2012. Essa população tinha $\mathrm{FE}$ média de $23 \%$ e a maioria apresentava classe NYHA III/IV. Os autores encontraram melhora em longo prazo na qualidade de vida, NYHA, FE e medidas de pró-BNP no seguimento desses pacientes. A curva de sobrevida apresentou diminuição expressiva da mortalidade quando comparada ao modelo de predição de mortalidade na IC pela Meta-analysis Global Group in Chronic Heart Failure (MAGGIC) - 13,1\% versus $18,4 \%$ no primeiro ano e $32,1 \%$ versus $40 \%$ no terceiro ano.

Recente estudo, conduzido por Liu et al. ${ }^{20}$, avaliou os efeitos dessa terapia em 41 pacientes com $\mathrm{FE}<40 \%$. O seguimento foi de seis anos e os casos foram comparados 1:1 com controles, com perfil semelhante de idade, FE, medicação e causa da ICC. O endpoint primário foi todas as causas de mortalidade e os endpoints secundários incluíram internação por IC e morte cardiovascular. A FE foi de $28 \%$ e todas as causas de mortalidade foram inferiores no grupo MCC. Quando estratificado pela FE, pacientes com abaixo de $25 \%$ não demonstraram melhora significativa na mortalidade. No entanto, na população com FE entre 25 e 40\%, a diminuição da mortalidade foi expressiva no grupo MCC. Melhora similar foi encontrada nos endpoints secundários.

Kloppe et al. ${ }^{21}$ acompanharam, por 4,5 anos, 68 pacientes submetidos a implante de MCC e com FE média de 26\% em dois centros na Alemanha. Demonstrou-se a diminuição da mortalidade no ano 1, 2 e 5 no grupo MCC comparado a modelo de previsão de mortalidade de SHFM.

\section{Perspectivas Futuras}

Não obstante o grande potencial relacionado a essa nova terapia, uma série de desafios ainda devem ser superados para que se possa adicioná-la ao atual arsenal terapêutico na ICC.
Grande parte dos pacientes com indicação para MCC, atual, baseada nas diretrizes europeias22 e estudos clínicos, já o é com dispositivos cardíacos como TRC e CDI. Isso significa que esses já têm um, dois, três ou mais caboseletrodos dentro do coração. Como essa terapia, no estado da arte atual, requer o implante de no mínimo três caboseletrodos, muitos problemas relacionados ao excesso de cabos, como trombose e aumento de risco de infecção, podem surgir. Há estudos de aprimoramento dessa terapia, como, por exemplo, acoplamento de um cardiodesfibrilador ao mesmo dispositivo do MCC.

Outra limitação atual é a necessidade de detecção de onda P para liberação do impulso pelo MCC que impede que pacientes com fibrilação atrial e ectopias frequentes se candidatem ao uso desse dispositivo. Melhorias no algoritmo, evitando a necessidade da sincronização com a onda $\mathrm{P}$, permitiria que esses pacientes também pudessem fazer uso dessa terapia, assim como evitaria a utilização de um cabo-eletrodo no átrio.

Embora a melhora em parâmetros de qualidade de vida e capacidade funcional seja importante, ainda falta demonstrar, com grandes estudos randomizados, duplo- cegos e multicêntricos, o impacto sobre a sobrevida e a melhora da mortalidade desses pacientes para mudar paradigmas em relação a essa nova terapia.

Atualmente, as diretrizes europeias para tratamento de pacientes com IC consideram aqueles com disfunção ventricular, CF II-III e QRS < 120 ms possíveis candidatos ao uso dessa nova tecnologia22.

\section{CONTRIBUIÇÃO DOS AUTORES}

Conceitualização, Salgado C; Investigação, Silvestrini TL; Redação, Silvestrini TL; Revisão, Ronsoni; Supervisão, Salgado C.

\section{REFERÊNCIAS}

1. Epstein AE, DiMarco JP, Ellenbogen KA, Estes NAM, Freedman RA, Gettes LS, et al. ACC/AHA/HRS 2008 Guidelines for Device-Based Therapy of Cardiac Rhythm Abnormalities: a report of the American College of Cardiology/American Heart Association Task Force on Practice Guidelines (Writing Committee to Revise the ACC/AHA/NASPE 2002 Guideline Update for Implantation of Cardiac Pacemakers and Antiarrhythmic Devices) developed in collaboration with the American Association for Thoracic Surgery and Society of Thoracic Surgeons. J Am Coll Cardiol. 2008;51(21):e1-62. https://doi.org/10.1016/j.jacc.2008.02.032

2. Wilcox JE, Fonarow GC, Zhang Y, Albert NM, Curtis AB, Yancy CW et al. Clinical effectiveness of cardiac resynchronization and implantable cardioverter-defibrillator therapy in men and women with heart failure: findings from IMPROVE HF. Circ Heart Fail. 2014;7(1):146-53. https://doi.org/10.1161/ CIRCHEARTFAILURE.113.000789 
3. Abi-Samar F, Gutterman D. Cardiac contractility modulation: a novel approach for the treatment of heart failure. Heart Fail Rev. 2016;21(6):645-60. https://doi. org/10.1007\%2Fs10741-016-9571-6

4. Braunwald E, Zipes DP, Libby P, Bonow RO. Braunwald's hearth disease: a text book of cardiovascular medicine. $7 a$ ed. 2015.

5. Dibb KM, Graham HK, Venetucci LA, Eisner DA, Trafford AW. Analysis of cellular calcium fluxes in cardiac muscle to understand calcium homeostasis in the heart. Cell Calcium. 2007;42(4-5):503-512. https://doi.org/10.1016/j. ceca.2007.04.002

6. Bears DM. Cardiac excitation-contraction coupling. Nature. 2002;415(6868):198-205. https://doi.org/10.1038/415198a

7. Burkhoff D, Shemer I, Felzen B, Shimizu J, Mika Y, Dickstein $M$, et al. Electric currents applied during the refractory period can modulate cardiac contractility in vitro and in vivo. Heart Fail Rev. 2001;6(1):27-34.

8. Morita H, Suzuki G, Haddad W, Mika Y, Tanhehco EJ, Sharov VG, et al. Cardiac contractility modulation with nonexcitatory electric signals improves left ventricular function in dogs with chronic heart failure. J Card Fail. 2003;9(1):69-75. https://doi.org/10.1054/jcaf.2003.8

9. Morita H, Suzuki G, Haddad W, Mika Y, Tanhehco EJ, Goldstein S, et al. Long-term effects of non-excitatory cardiac contractility modulation electric signals on the progression of heart failure in dogs. Eur J Heart Fail. 2004;6(2):145-150 https://doi.org/10.1016/j.ejheart.2003.11.001

10. Butter C, Rastogi S, Minden HH, Meyhofer J, Burkhoff D, Sabbah HN. Cardiac contractility modulation electrical signals improve myocardial gene expression in patients with heart failure. J Am Coll Cardiol. 2008;51(18):1784-9. https:// doi.org/10.1016/j.jacc.2008.01.036

11. Imai M, Rastogi S, Gupta RC, Mishra S, Sharov VG, Stanley WC, et al. Therapy with cardiac contractility modulation electrical signals improves left ventricular function and remodeling in dogs with chronic heart failure. J Am Coll Cardiol. 2007;49(21):2120-8. https://doi.org/10.1016/j. jacc.2006.10.082

12. Lyon AR, Samara MA, Feldman DS. Cardiac contractility modulation therapy in advanced systolic heart failure. Nat Rev Cardiol. 2013;10(10):584-98. https://doi.org/10.1038/ nrcardio.2013.114

13. Stix $G$, Borggrefe $M$, Wolpert $C$, Hindricks $G$, Kottkamp $H$, Bocker D, et al. Chronic electrical stimulation during the absolute refractory period of the myocardium improves severe heart failure. Eur Heart J. 2004;25(8):650-5. https:// doi.org/10.1016/j.ehj.2004.02.027

14. Borggrefe MM, Lawo $T$, Butter C, Schmidinger H, Lunati M, Pieske B, et al. Randomized, double blind study of nonexcitatory, cardiac contractility modulation electrical impulses for symptomatic heart failure. Eur Heart J. 2008;29(8):101928. https://doi.org/10.1093/eurheartj/ehn020

15. Kadish A, Nademanee K, Volosin K, Krueger S, Neelagaru $\mathrm{S}$, Raval $\mathrm{N}$, et al. A randomized controlled trial evaluating the safety and efficacy of cardiac contractility modulation in advanced heart failure. Am Heart J. 2011;161(2):329-37. https://doi.org/10.1016/j.ahj.2010.10.025

16. Kwong JS, Sanderson JE, Yu CM. Cardiac contractility modulation for heart failure: a meta-analysis of randomized controlled trials. Pacing Clin electrophysio. I. 2012;35(9):1111 8. https://doi.org/10.1111/j.1540-8159.2012.03449.x

17. Giallauria F, Vigorito C, Piepoli MF, Stewart Coats AJ. Effects of cardiac contractility modulation by non-excitatory electrical stimulation on exercise capacity and quality of life: an individual patient's data meta-analysis of randomized controlled trials. Int J Cardiol. 2014;175(2):352-7. https://doi. org/10.1016/j.ijcard.2014.06.005

18. Schau T, Seifert M, Meyhofer J, Neuss M, Butter C. Long-term outcome of cardiac contractility modulation in patients with severe congestive heart failure. Europace. 2011;13(10):143644. https://doi.org/10.1093/europace/eur153

19. Kuschyk J, Roeger S, Schneider R, Streitner F, Stach K, Rudic B, et al. Efficacy and survival in patients with cardiac contractility modulation: long-term single center experience in 81 patients. Int J Cardiol. 2015;183(15):76-81. https://doi. org/10.1016/j.ijcard.2014.12.178

20. Liu M, Fang F, Luo XX, Shlomo BH, Burkhoff D, Chan JY, et al. Improvement of long-term survival by cardiac contractility modulation in heart failure patients: a case-control study. Int J Cardiol. 2016;206(1):122-6. https://doi.org/10.1016/j. ijcard.2016.01.071

21. Kloppe A, Lawo T, Mijic D, Schiedat F, Muegge A, Lemke B. Long-term survival with cardiac contractility modulation in patients with NYHA II or III. Int J Cardiol. 2016; 209 (15):291295 https://doi.org/10.1016/j.ijcard.2016.02.001

22. Ponikowiski P, Voors AA, Anker SD, Bueno H, Cleland JGF, Coats AJS et al. 2016 ESC Guidelines for the diagnosis and treatment of acute and chronic heart failure: The Task Force for the diagnosis and treatment of acute and chronic heart failure of the European Society of Cardiology (ESC). Eur Heart J. 2016;37(27):2129-2200. https://doi.org/10.1093/ eurheartj/ehw128 